\title{
MODELAMIENTO Y EVALUCION DE UNA CONTRACCIÓN DE UN TÚNEL DE VIENTO SÚBSONICO
}

\section{MODELING AND EVALUATION OF A CONTRACTION OF A SUBSONIC WIND TUNNEL}

\author{
Ing. Juan Javier Mora González*, PhD. Juan Carlos Serrano Rico**, \\ MSc. Edison Martínez Oviedo**. \\ * Universidad de Pamplona, Facultad de Ingenierías y Arquitectura, Programa de \\ Ingeniería Mecánica. \\ Km 1 vía Bucaramanga, Pamplona, Norte de Santander, Colombia. \\ E-mail: moragonzalezj@gmail.com. \\ ** Universidad de Pamplona, Facultad de Ingenierías y Arquitectura, Programa de \\ Ingeniería Mecánica. \\ Km 1 vía Bucaramanga, Pamplona, Norte de Santander, Colombia. \\ E-mail: $\{$ jcserrano, edisonmartinez $\} @$ unipamplona.edu.co.
}

Resumen: Este trabajo presenta el modelamiento y evaluación de una contracción para un túnel de viento subsónico. Las simulaciones se realizaron a tres diferentes formas de contracción comúnmente utilizadas en túneles de viento, lo cual permitió a través de software tipo CAE analizar la uniformidad del fluido, turbulencia y el coeficiente de presión de a lo largo de la contracción. Estas simulaciones demuestran que la contracción propuestas por (Whitehead, Wu, \& Waters, 1951) presenta un mejor desempeño en cuanto a las variables estudiadas. Con el objetivo de mejorar la uniformidad del fluido y disminuir su turbulencia en la salida de la contracción, fue utilizado un trip en la región de entrada de la contracción; lo cual mejoró en un 92,4\% el coeficiente de presión y la intensidad de turbulencia.

Palabras clave: Contracción, capa límite, uniformidad, coeficiente de presión.

\begin{abstract}
This paper presents the modeling and evaluation of a contraction for a subsonic wind tunnel. The simulations were made to three different shapes of contraction commonly used in wind tunnels, which allowed CAE type software to analyze the uniformity of the fluid, turbulence and the pressure coefficient along the contraction. These simulations show that the contraction proposed by (Whitehead et al., 1951) presents a better performance in terms of the variables studied. In order to improve the uniformity of the fluid and decrease its turbulence at the exit of the contraction, a trip was used in the region of the contraction input; which improved the pressure coefficient and turbulence intensity by $92.4 \%$.
\end{abstract}

Keywords: Contraction, boundary layer, uniformity, pressure coefficient. 


\section{INTRODUCCIÓN}

La utilización de energías renovables dentro de la matriz energética de los países está teniendo más importancia. La energía eólica constituye en la fuente energética de mayor penetración y de investigaciones en diferentes campos. Como por ejemplo, el estudio posibles lugares para el desarrollo de proyectos eólicos como los evaluados por (Leal González \& Herández Cely, 2013) y (Álvarez Castañeda, Alvarado Fajardo, \& Cardona, 2014). Investigaciones en aerogeneradores tiene un capítulo relevante en la energía eólica para lo cual el uso de túneles de viento constituye instrumento principal para realizar diversos análisis del comportamiento de los flujos sobre los cuerpos. En él se generan corrientes de aires el cual simulan distintas fuerzas, fenómenos aerodinámicos, entre otros. Estos experimentos dentro de los túneles de viento con condiciones controladas para ciertas condiciones como uniformidad de flujo e intensidad de turbulencia.

Hay muchos tipos de túneles de viento y se pueden clasificar por ejemplo, velocidad de flujo dividiéndolos en cuatro grupos.

Túneles de viento subsónico o de baja velocidad Túneles de viento transónicos

Túneles de viento supersónico

Túneles de viento hipersónico

Los túneles de viento subsónico o de baja velocidad son el tipo más común y el túnel de viento el cual será proyectado la contracción es de este tipo. Los túneles de viento Transónicos son comunes en la industria aeronáutica, ya que la mayoría de los aviones comerciales operan en este régimen. Los túneles de viento supersónicos pueden usarse para investigar el comportamiento de motores jet y aviones militares. Los túneles de viento hipersónicos encuentran sus aplicaciones en cohetes y vehículos espaciales. Otra forma de categorizar los túneles de baja velocidad está dividiéndolos en túneles de viento de circuito abierto o de circuito cerrado. En los túneles de viento de circuito abierto, la potencia necesaria para utilizar el túnel de viento es alta debido a la pérdida de energía por el aire de recambio. Los túneles de viento de circuito cerrado recirculan el aire y por lo tanto necesitan menos potencia para lograr una velocidad de flujo dada (Lindgren \& Johansson, 2002).
El diseño de la contracción del túnel de viento es extremadamente importante para el suministro del flujo de prueba con calidad en la sección de trabajo. La contracción es necesaria para dos propósitos principales. En primer lugar, aumentar la velocidad media del flujo de ensayo de modo que los dispositivos mejoradores de la calidad del flujo (malla, colmena) actúen en un entorno de baja velocidad en donde se reducen las pérdidas de presión. En segundo lugar, una contracción reduce las variaciones de velocidad media y fluctuante a una fracción más pequeña de la velocidad media. El diseño de una contracción requiere que el flujo permanezca unido a lo largo de la longitud de la contracción y minimice la altura de la capa límite de salida así como la no uniformidad del flujo de salida (Doolan \& Morgans, 2007).

Sin embargo, para las contracciones de longitud finita existen dos regiones de gradiente de presión adversa, uno cerca de la entrada y otro en la región de salida. Como resultado, el perfil de velocidad a través del plano de salida de una contracción es no uniforme que conduce a una reducción de la longitud útil de la sección de trabajo. Además, las dos regiones de presión adversa pueden provocar una separación intermitente a gran escala de la capa límite que da lugar a una mayor inestabilidad del flujo en la sección de trabajo (Watmuff, 1986).

Sin una contracción bien diseñada, la calidad del flujo en la sección de prueba será deficiente. Los fines de la contracción son: Disminuir las perturbaciones de flujo procedentes de la cámara de sedimentación, acelerar el flujo, evitar separación de la capa límite y crear un perfil de velocidad uniforme que entra en la sección de prueba. El parámetro más importante que determina la magnitud de estos efectos es la relación de contracción, CR. El desafío en diseño de contracción es encontrar una contracción lo más pequeña posible sin separación y con una buena calidad de flujo de salida (Hjärne, Löfdahl, \& Larsson, 2003).

\section{METODOLOGIA}

\subsection{Selección de la forma de contracción}

Mediante la búsqueda bibliográfica de los perfiles de contracción, se hallaron tres formas utilizadas para la construcción de los túneles de viento de baja velocidad, las cuales aportan unas características de flujo cerca de la pared y en la 
salida de la geometría dada. La figura 1, representa los contornos de la geometría de cada una de las contracciones seleccionada previamente. Cabe resaltar que las relaciones de contracción y la longitud se colocaron fijas para las simulaciones elaboradas en el software de ANSYS.

Para definir o formular los criterios de diseño, una vez que se elige la relación de contracción, los criterios de diseño en el diseño de contracción del túnel de viento son: uniformidad del flujo de salida, separación, grosor de la capa límite de salida y espacio/costo. Los parámetros de diseño son la longitud, la forma de la pared y el número de Reynolds. De estos cuatro criterios, los más importantes son los dos primeros. Por lo tanto, el procedimiento de diseño propuesto aquí está dirigido principalmente a evitar la separación y producir el perfil de velocidad de salida con una no uniformidad prescrita.

La primera forma de contracción fue determinada por el polinomio de 5to orden sugerido por (Bell \& Mehta, 1988):

$$
Y(X)=H i-(H i-H e)\left[6\left(X^{\prime}\right) 5-15\left(X^{\prime}\right) 4+\right.
$$$$
\left.10\left(X^{\prime}\right) 3\right]
$$

Donde $H i$ es la altura de entrada del flujo de aire de la contracción, $\mathrm{He}$ es la altura de salida de flujo y $X$ es la longitud de dirección de flujo y $L$ la longitud de la contracción.

La segunda forma de pared de contracción fue determinada por polinomio cubico coincidente sugerido por (Morel, 1977):

$$
\begin{aligned}
& Y(X)=\left(H_{1}-H_{2}\right)\left[1-\frac{1}{X^{2}}\left(\frac{x}{L}\right)^{3}\right]+ \\
& H_{2} \quad \frac{x}{L} \leq X \\
& Y(X)=\frac{\left(H_{1}-H_{2}\right)}{(1-X)^{2}}\left(1-\frac{x}{L}\right)^{3}+H_{2} \\
& \frac{x}{L}>X
\end{aligned}
$$

Los coeficientes de presión en la entrada $\left(C p_{i}\right)$ y salida $\left(C p_{e}\right)$ de la contracción se definen en las ecuaciones 5 y 6 . En donde los subíndices $i$ y $e$ se refieren a los puntos de velocidad máxima y mínima en la pared, $U 1, \infty, U 2,_{\infty}$ es la velocidad de corriente libre en la entrada y salida de la contracción (Morel, 1975).

$$
\begin{aligned}
& C_{p i}=1-\left(\frac{u_{i}}{U_{1, \infty}}\right)^{2} \\
& C_{p e}=1-\left(\frac{U_{2, \infty}}{u_{e}}\right)^{2}
\end{aligned}
$$

La tercera forma de pared de contracción fue determinada por una función potencial sugerida por (Whitehead et al., 1951):

$$
\begin{aligned}
& a_{C}-a_{B}=\frac{2 U k}{\pi} \log \frac{[R-b]\left[R^{2}+a^{2}\right]}{a\left[(R-b)^{2}+R^{2}\right]} \\
& a_{C}-a_{B}=\frac{2 U k}{\pi} \log \frac{\left[R^{2}-a^{2}\right]\left[R^{2}+(R-b)^{2}\right]}{\left[R^{2}+a^{2}\right]\left[R^{2}-(R-b)^{2}\right]}
\end{aligned}
$$

Donde $U$ es la velocidad, $k$ es la relación de contracción en dos dimensiones, $R$ es el radio de circulo en el plano q, $a$ es la longitud $A B$ y $b$ es la longitud $D E$.

En la figura 1, se observan las diferentes geometrías de las contracciones objeto de estudio; las contracciones tienen la misma relación de contracción [CR] de 7 y la misma longitud, la cual es de $3,5[\mathrm{~m}]$.

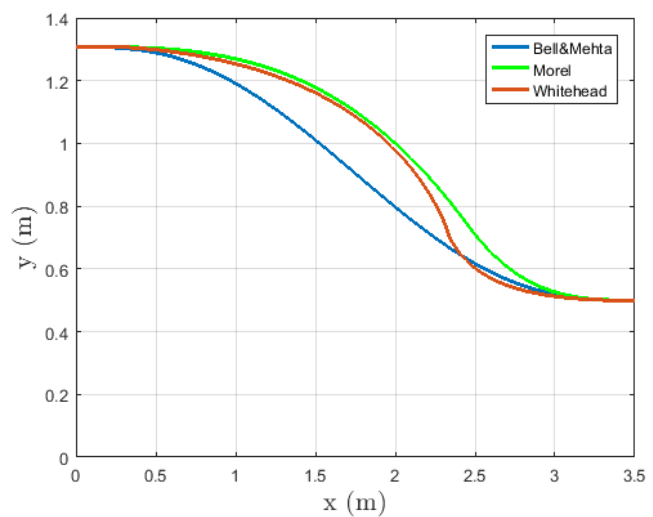

Figura 1. Perfiles de contracción de los túneles de viento. Fuente: Autor.

\subsection{Criterio de calidad de diseño de la contracción}

Para contrastar las diferentes formas de contornos de las contracciones utilizadas, debemos tener en cuentas las variables que afectan el flujo dentro de la geometría el cual son los factores importantes 
para que en la sección de prueba haya calidad en el flujo.

(Dehghan Manshadi, Mirzaei, Soltani, \& Ghorbanian, 2008) Menciona que este fenómeno se origina en la entrada de la contracción porque la pared cambia repentinamente de una región plana a una curva, por lo que la línea de flujo cercana a la pared se acumularía y eventualmente aumentaría la presión relativa en la región.

El coeficiente de presión tiene un máximo y un mínimo para la distribución de presión estática a lo largo de la pared en dos lugares cerca de la entrada y la salida, respectivamente, lo que resulta en regiones de gradientes de presión adversos. La separación puede ocurrir en las regiones de gradiente de presión adversa, por lo que la contracción debe diseñarse para minimizar la posibilidad de separación (Mathew, Bahr, Carroll, Sheplak, \& Cattafesta, 2006).

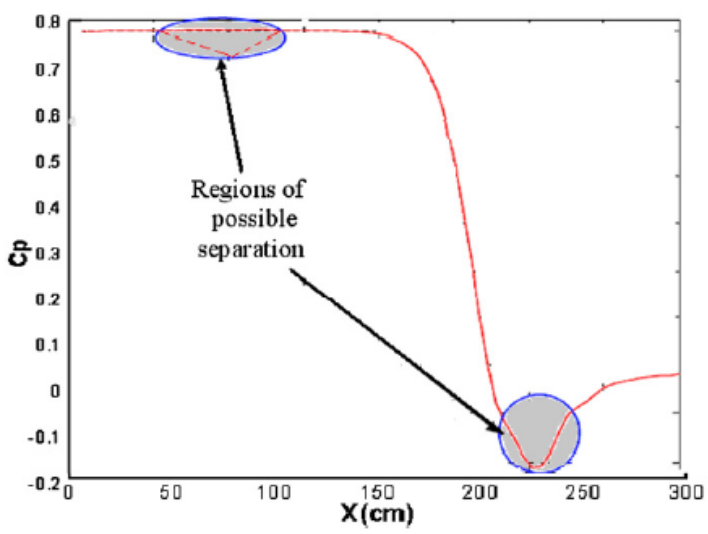

Figura 2. Ejemplo de distribución de presión en la pared en un túnel de viento. Fuente: (Ghorbanian, Soltani, \& Manshadi, 2011).

Para obtener resultados medidos y calculados regulados, se utilizará un parámetro adimensional

$C_{p}=\frac{P_{(x)}-P_{e}}{0.5 * \rho * U_{e}^{2}}$

Donde,

$U \quad$ Componente $\mathrm{x}$ de la velocidad fuera de la capa límite (paralela a la superficie solida), [m/s]

$\rho \quad$ Densidad del fluido, $\left[\mathrm{kg} / \mathrm{m}^{3}\right]$

$P_{(x)}$ y $P_{(e)}$ Presión absoluta o manométrica $[\mathrm{Pa}]$

La uniformidad de flujo a través de las simulaciones en el plano de salida se obtendrá campos de velocidad constantes.
(Kolmogorov, 1941) fundamenta su teoría clásica de turbulencia, de mutua semejanza y plantea que la energía cinética $k$ de los remolinos de mayor escala fluye hacia los de menor escala a través de un mecanismo de cascada. Además, esa energía fluyente es constante entre las diferentes escalas longitudinales de los remolinos y está dada por el coeficiente de disipación $\varepsilon$ de la energía cinética de $k$.

\subsection{Trips en la capa límite: Metodología de optimización}

Los dispositivos de transición son utilizados para perturbar el estado de capa límite alrededor o sobre un objeto de estudio. Estos los podemos encontrar dentro de un túnel viento para desarrollar investigaciones, en consecuencia se crean perturbaciones a una distancia más corta de donde probablemente se producen. Estos dispositivos son útiles, porque los espacios para la instalación de un túnel de viento pueden ser limitados.

En planeadores, estos dispositivos (strips) son utilizados para crear una capa limite turbulenta con el fin de postergar la separación que causaría un aumento sustancial de resistencia. Las configuraciones utilizadas comúnmente son rugosidad, alambre circular, paso superficial lo cual son formas muy simples (Slangen, 2009).

Se encontraron varios métodos para energizar la capa límite dentro de un túnel de viento pero se utilizó la forma de alambre circular por su forma sencilla de aplicación.

\section{RESULTADOS Y DISCUSIÓN}

En la etapa de análisis de resultados se tiene en cuenta cada uno de los objetivos de este estudio. A continuación se lleva a cabo la forma del análisis de estudio.

\subsection{Parámetros}

A manera de poder obtener resultados dentro del software, las propiedades añadidas de las condiciones a la que el fluido fue empleado dentro de las simulaciones fueron las siguientes:

Tabla 1. Condiciones del aire a $20^{\circ}$ Celsius. Fuente:(Cengel \& Cimbala, 2012).

Datos de las condiciones del fluido 


\begin{tabular}{|c|c|}
\hline Densidad & {$\left[1.204 \mathrm{~kg} / \mathrm{m}^{3}\right]$} \\
\hline Calor específico, $\mathrm{C}_{\mathrm{p}}$ & {$[1007 \mathrm{~J} / \mathrm{kg} * \mathrm{~K}]$} \\
\hline Conductividad térmica, $\mathrm{k}$ & {$[0.02476 \mathrm{~W} / \mathrm{m} * \mathrm{~K}]$} \\
\hline Viscosidad dinámica, $\mu$ & $1.825 \times 10^{-5}\left[\mathrm{~kg} / \mathrm{m}^{*} \mathrm{~s}\right]$ \\
\hline Masa molar & $28.97[\mathrm{~kg} / \mathrm{kmol}]$ \\
\hline Velocidad, $v(x)$ & $8.571[\mathrm{~m} / \mathrm{s}]$ \\
\hline Velocidad, $v(y)$ & $0.1[\mathrm{~m} / \mathrm{s}]$ \\
\hline Velocidad, $v(z)$ & $0.1[\mathrm{~m} / \mathrm{s}]$ \\
\hline Presión, $\mathrm{P}$ & $101.325[\mathrm{kPa}]$ \\
\hline Salida de presión & $0[\mathrm{~Pa}]$ \\
\hline
\end{tabular}

La relación de contracción utilizada para para las diferentes configuraciones basado en el anterior parámetro fue de 7 , lo cual permite desarrollarla en un espacio limitado la construcción del túnel viento. La velocidad requerida a la salida de la contracción es de $60 \mathrm{~m} / \mathrm{s}$.

Para los parámetros del mallado ANSYS lo genera de forma uniforme en el ensamblado de estudio, el mallado utilizado ha sido el extra fino (Leifsson \& Koziel, 2015) donde las contracciones son de secciones cuadradas y el número de elementos alcanzados para la simulación está en 2.503 .000 (sección de salida de la contracción: $1\left[\mathrm{~m}^{2}\right]$ y la sección de entrada de la contracción: $6.8434\left[\mathrm{~m}^{2}\right]$ ).

Para los criterios de convergencia se toma lo que se obtenga al haber ocurrido en programa de solución que es un objetivo residual en seis órdenes de magnitud o el número máximo de iteraciones, que para este fueron de 1000 .

La solución de las constantes de la ecuación de Navier-Stokes a través del software de dinámica computacional de fluidos (Doolan \& Morgans) CFX, está en confluencia con la estructura del mallado y modelo de turbulencia Shear Stress Transport (SST).

A través de la búsqueda bibliográfica observando el modelo más adecuado para implementar dentro del desarrollo de la simulación, ((Leifsson \& Koziel, 2015) supone que el flujo es de estado estable, incompresible y turbulento. La descripción del fluido es aire y sus cualidades son tomadas de tablas. El modelo más adecuado es el (SST) de (Menter et al., 2003), este modelo de turbulencia seleccionado fue por la irregularidad de la forma de la contracción y para problemas aerodinámicos donde se involucren capa límite bajo gradientes de presión adversa y separación.

\subsection{Resultados numéricos}

En esta fase de análisis de resultados tomamos en cuenta cada uno de los objetivos planteados anteriormente. A continuación presentamos la forma de análisis:

\subsubsection{Uniformidad de flujo}

Se investigaron tres formas de pared para contracciones de túneles de viento, se estudió la uniformidad de flujo y coeficiente de presión. Debido a la relación de contracción que fue aplicado a cada uno de los perfiles, apreciamos una tendencia de velocidades que va aumentado a través de las paredes similares en la salida de la contracción. Mostrando los diferentes perfiles, consideramos una diferencia del flujo procedente de la cámara de ajuste, la velocidad en la contracción de (Whitehead, Wu, \& Waters, 1951) (fig. 5) presenta una uniformidad de flujo con anterioridad en comparación con las contracciones de (Bell \& Mehta, 1988) (fig. 3) y (Morel, 1977) (fig.4).

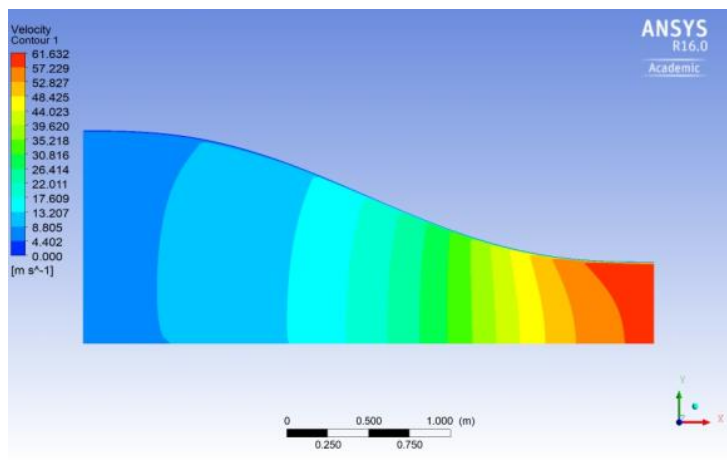

Figura 3. Velocidad en la contracción (Bell \& Mehta, 1988). Velocidad de salida $60 \mathrm{~m} / \mathrm{s}$.

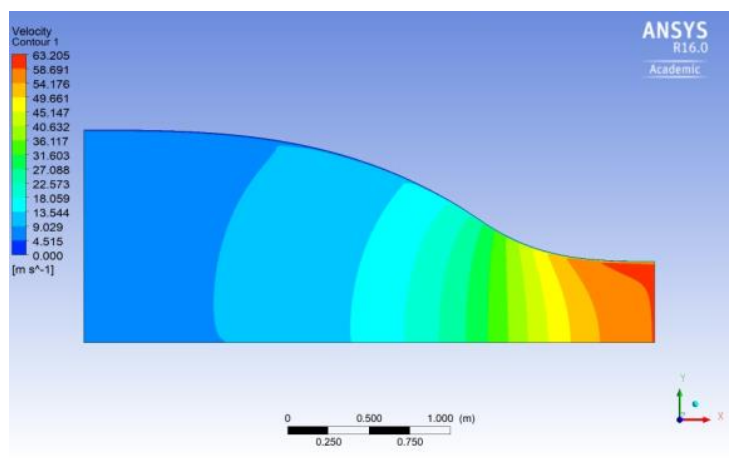

Figura 4. Velocidad en la contracción (Morel, 1977). Velocidad de salida $60 \mathrm{~m} / \mathrm{s}$. 


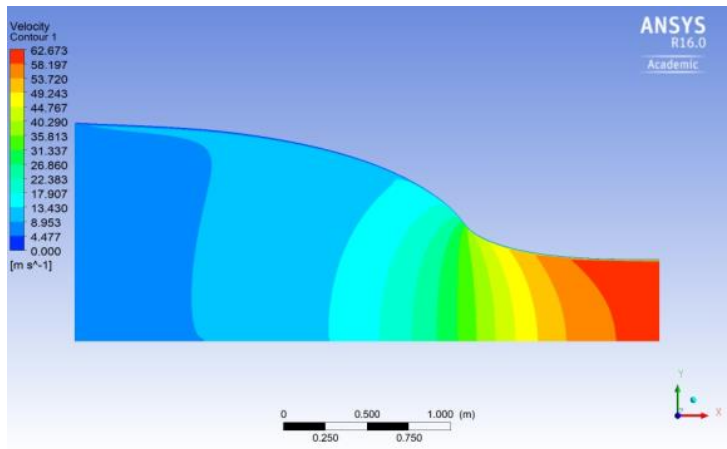

Figura 5. Velocidad en la contracción (Whitehead et al., 1951). Velocidad de salida $60 \mathrm{~m} / \mathrm{s}$.

Como el diseño de la contracción tuvo el fin de lograr la uniformidad en las corrientes libres de la velocidad y las fluctuaciones de la turbulencia, en las figs. 3-5, visualizamos que la velocidad de flujo en toda el área de salida es uniforme, donde la delgada capa límite paralela a la pared afecta de manera mínima este flujo. También se puede observar que la contracción de Whitehead presenta uniformidad de flujo muchos antes que las otras contracciones a una distancia $\mathrm{x}=3,233$ [m] de la entrada de la contracción.

\subsection{Coeficiente de presión longitudinal}

A medida que el flujo recorre la contracción concibe un parámetro adimensional que permite a cada una de las contracciones medir en qué parte de su geometría se localiza un gradiente de presión alto, esto significa que puede haber separación de la capa límite. La siguiente gráfica se pude divisar los diferentes gradientes de presión, donde queda claro que la contracción Whitehead sigue siendo la mejor aplicación de diseño para un túnel de viento que necesite uniformidad en su flujo de salida en comparación con sus contracciones homólogas (Bell \& Mehta, 1988) y (Morel, 1977).

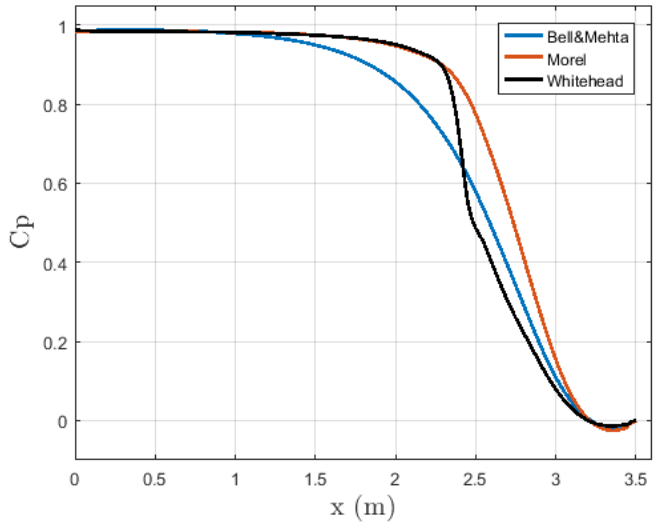

Figura 6. Comparación de los coeficientes de presión. Fuente: Autor.

\subsection{Intensidad de Turbulencia}

La intensidad de turbulencia generada en cada una de las formas de contracción crea vibraciones o ruido dentro del túnel de viento, en las siguientes gráficas se observó que cada una de las formas de pared en la zona donde se harán las pruebas de mediciones, el nivel de turbulencia es nulo, lo que permite la calidad de flujo en la cámara de ensayos. En las figs. 7-9 se observó los diferentes rendimientos:

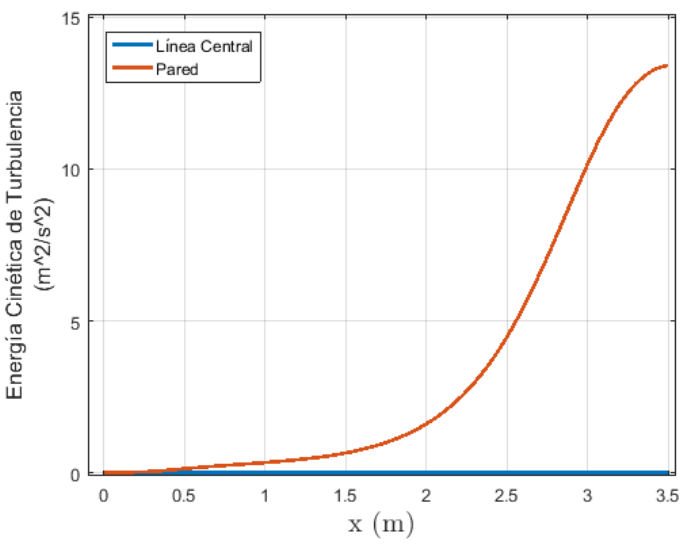

Figura 7. Intensidad de turbulencia. Contracción Bell\&Mehta.

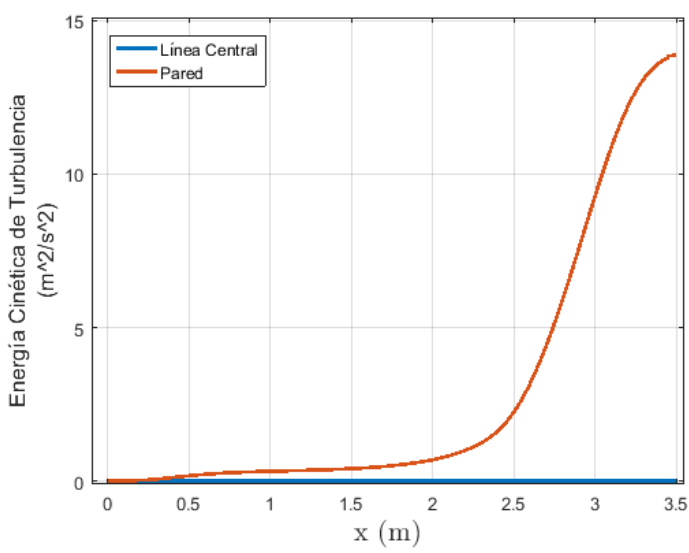

Figura 8. Intensidad de turbulencia. Contracción Morel. 


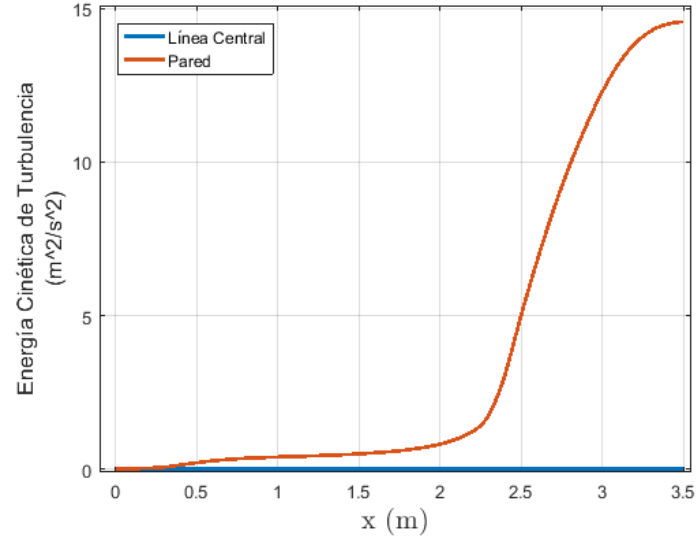

Figura 9. . Intensidad de turbulencia. Contracción Whitehead.

\subsection{Optimización}

Como ya se ha mencionado anteriormente, la condición esencial dentro de la contracción es disminuir el gradiente de presión adverso, logrado esto se causa grandes beneficios con respecto a la separación de la capa límite adyacente a la pared en la entrada y la salida de la contracción.

Se planteó colocar trip strips dentro de la contracción para modificar la capa límite y así potenciar la capa limite cerca de las paredes. Generar una capa límite turbulenta con anticipación genera un retraso en la separación del flujo. Para los ensayos realizados se escogieron tres posiciones internamente, donde se colocó la configuración circular de $2 \mathrm{~mm}$ de diámetro, las distancias son con respecto a la entrada de: $x=70 ; 90 ; 110[\mathrm{~cm}]$.

\subsubsection{Resultados numéricos de coeficiente de presión.}

Como se mencionó anteriormente, la contracción elegida por sus resultados obtenidos fue la forma de pared Whitehead, ya que este aportó valores positivos en la uniformidad de flujo y un coeficiente de presión más bajo en comparación con las otras, lo que generó menos separación en la capa límite del fluido. Al añadir estos dispositivos de transición dentro de la contracción elegida, en la Fig. 11 se pudo ver que la configuración colocada a $90[\mathrm{~cm}]$ tuvo una disminución en el coeficiente de presión y gradiente de presión adverso, esto permite generar una capa limite turbulenta con anticipación lo que hace que la separación en el flujo se retrase. La tabla 2 y las Figs. 10-12, hacen referencia de los incrementos y decrementos con respecto a los valores de la contracción que no presenta modificaciones:

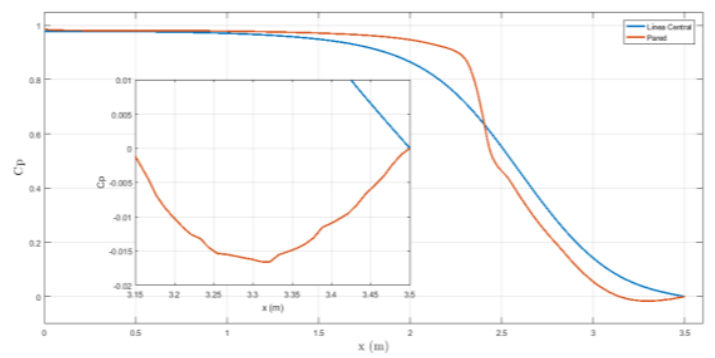

Figura 10. Coeficiente de presión. Trip strips 70 [cm]. Fuente: Autor.

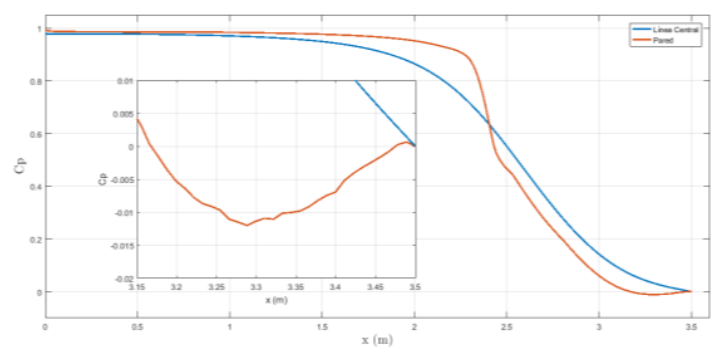

Figura 11. Coeficiente de presión. Trip strips 90 [cm]. Fuente: Autor.

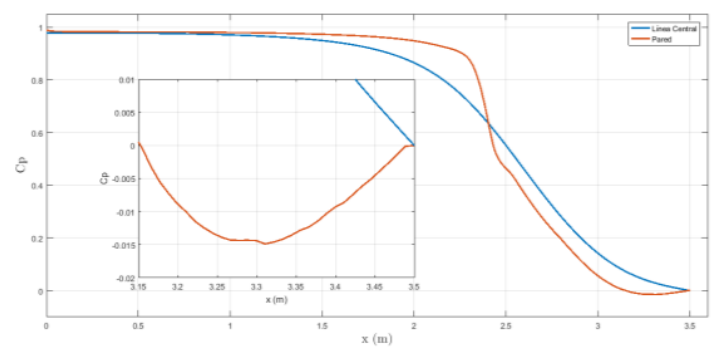

Figura 12. Coeficiente de presión. Trip strips 110 [cm]. Fuente: Autor.

En la tabla 2. Muestra el valor mínimo del coeficiente de presión obtenido en la región de salida de la contracción, se puede observar que cuando se ubica la trip strip a $90[\mathrm{~cm}]$, la separación de la capa límite tiene el mayor coeficiente de presión en comparación a las otras estudiadas.

Tabla 2. Incremento del coeficiente de presión con respecto al modelo base.

\begin{tabular}{|c|c|}
\hline Contracción & Coeficiente de presión \\
\hline Contracción sin trip strip & $-0,0136$ \\
\hline Optimización $70[\mathrm{~cm}]$ & $-0,0166$ \\
\hline
\end{tabular}




\begin{tabular}{|c|c|}
\hline Optimización $90[\mathrm{~cm}]$ & $-0,0120$ \\
\hline Optimización $110[\mathrm{~cm}]$ & $-0,0149$ \\
\hline
\end{tabular}

\section{PORCENTAJE (\%)}

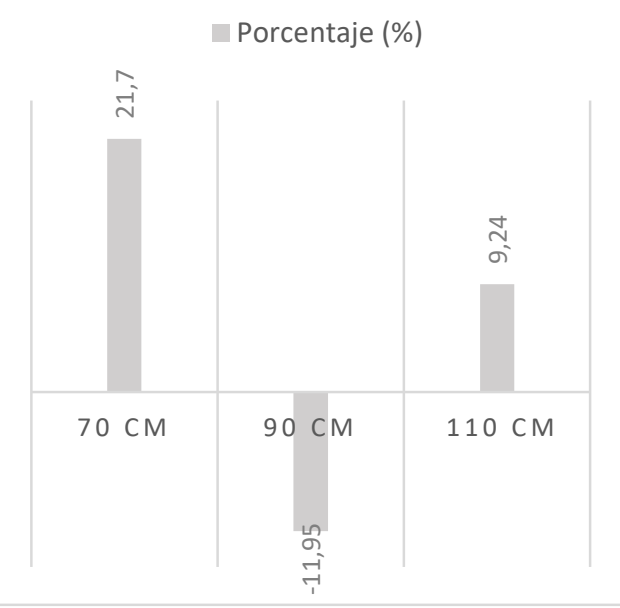

Figura 13. Comparación de porcentaje de Error entre las contracciones con respecto a la no modificada.

\subsubsection{Resultados numéricos de turbulencia.}

Los resultados obtenidos de intensidad de turbulencia de las optimizaciones generaron niveles más bajo con respecto a la contracción sin modificaciones interiores, lo que permite que haya menos ruidos y vibraciones en el pared de las contracciones. En la línea central donde se realizaran las pruebas dentro del túnel de viento la intensidad de turbulencia es nula lo cual no va afectar las mediciones de flujo a diferentes objetos de estudio. En las siguientes figuras se observa la reducción con respecto a la contracción no alterada:

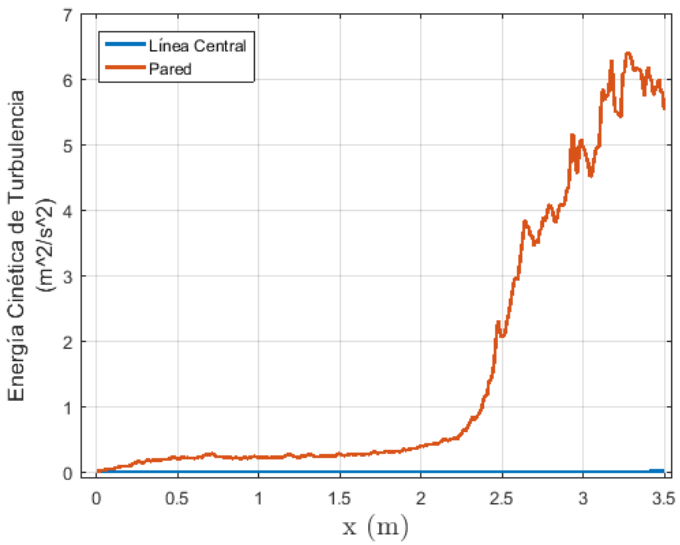

Figura 14. Intensidad de turbulencia. Contracción Whitehead optimización 1.

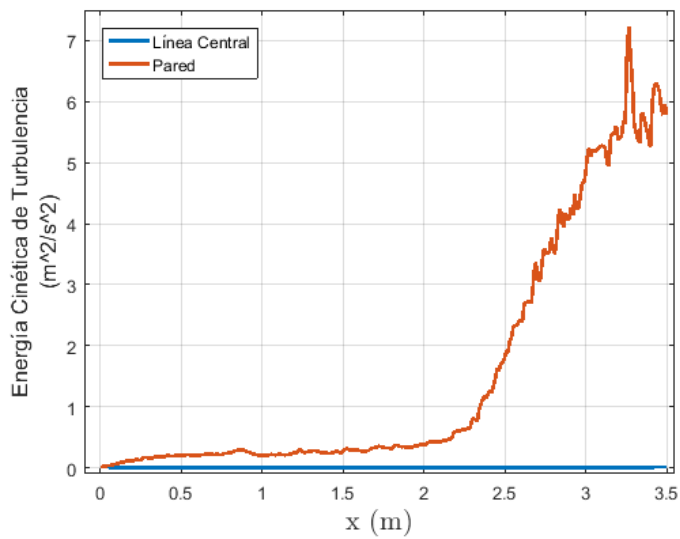

Figura 15. Intensidad de turbulencia. Contracción Whitehead optimización 2.

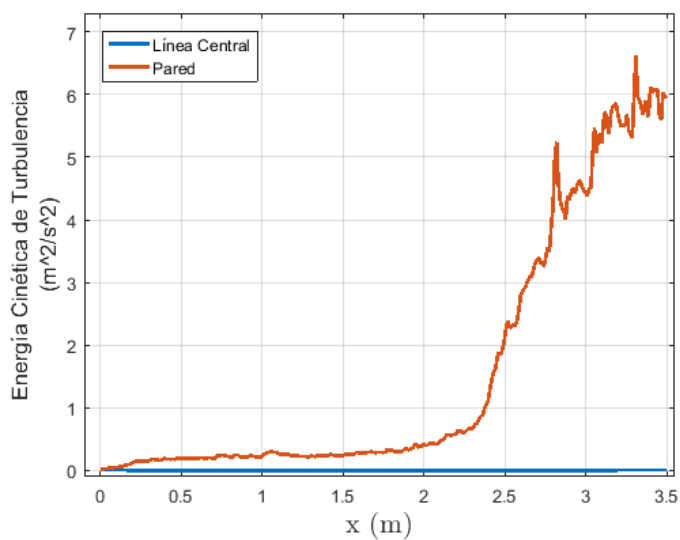

Figura 16. Intensidad de turbulencia. Contracción Whitehead optimización 3.

Tabla 3. Comparación de intensidad de turbulencia en la salida de la contracción.

\begin{tabular}{|c|c|}
\hline Contracción & $\begin{array}{c}\text { Intensidad de } \\
\text { Turbulencia }\left[\mathrm{m}^{2} / \mathrm{s}^{2}\right]\end{array}$ \\
\hline Whitehead sin trip strip & 14.5652 \\
\hline Optimización $70[\mathrm{~cm}]$ & 5.5283 \\
\hline Optimización $90[\mathrm{~cm}]$ & 5.7871 \\
\hline Optimización $110[\mathrm{~cm}]$ & 5.9919 \\
\hline
\end{tabular}

\section{CONCLUSIONES}

Los datos numéricos de las pruebas presentadas y discutidas determinaron las características del flujo en las tres formas de pared de las contracciones a través de las caras de entrada y salida, por medio 
de la simulación de los parámetros de flujo se posibilitó las siguientes conclusiones:

- Los resultados muestran que la uniformidad de la velocidad se cumple en las zonas de interés para las tres formas de pared, lo que obedece unos de los criterios de calidad de diseño para una contracción de túnel de viento de baja velocidad. La función potencial obtuvo mejores resultados con respecto al polinomio de 5to orden y al polinomio cúbico coincidente, este alcanzó la uniformidad de flujo de $92,4 \%$ con respecto al plano de entrada, lo que permite una calidad de flujo dentro del túnel de viento. La función potencial de (Whitehead et al., 1951) tiene un alto rendimiento de coeficiente de presión en comparación a sus semejantes, lo que permite que haya menos índice de separación de la capa límite. El polinomio de 5to orden y el polinomio cúbico coincidente superan por $41,18 \%$ y $86,76 \%$ con respecto a esta.

- Los distintos contornos de pared obtienen resultados muy positivos en relación a la intensidad de turbulencia, lo que atribuye en las regiones de prueba del túnel de viento no se presente.

- $\quad$ Las trip strips aplicada para modificar la geometría de la función potencial obtuvieron resultados que mejoraron el coeficiente de distribución de presión y la intensidad de turbulencia en la pared. A través de las simulaciones consideramos colocar estas modificaciones dentro del túnel de viento entre 70 y $110 \mathrm{~cm}$ de la sección de entrada de flujo, donde los mejores resultados fueron a $90 \mathrm{~cm}$ permitiendo un decrecimiento del $11,95 \%$ en el coeficiente de presión comparado con la contracción sin alteraciones de geometría, permitiendo energizar la capa límite y que no se presente separación en ella.

\section{RECONOCIMIENTOS}

Agradezco a la Universidad de Pamplona y cuerpo de docentes por darme la oportunidad de realizar este trabajo donde se deja planteado un estudio preliminar para el diseño de un túnel viento con fines académicos. La meta es seguir profundizando sobre este tema para lograr un desarrollo en diferentes aplicaciones donde se necesite dicho estudio.

\section{REFERENCIAS}

Álvarez Castañeda, W., Alvarado Fajardo, A., \& Cardona, C. (2014). Evaluación del potencial eólico en el Alto de Soracá -
Tunja. Revista Colombiana de Tecnologías de avanzada, 2(24), 90-92.

Bell, J. H., \& Mehta, R. D. (1988). Contraction design for small low-speed wind tunnels.

Cengel, Y. A., \& Cimbala, J. M. (2012). Mecánica de fluidos. Fundamentos y Aplicaciones. (S. A. d. C. V. McGrawHill/Interamericana editores Ed. segunda edicion ed.).

Dehghan Manshadi, M., Mirzaei, M., Soltani, M., \& Ghorbanian, K. (2008). Control of pressure gradient in the contraction of a wind tunnel. World Academy of Science, Eng. and Tech, 40, 261-266.

Doolan, C. J., \& Morgans, R. C. (2007). Numerical evaluation and optimization of low speed wind tunnel contractions. Paper presented at the 18th AIAA CFD Conference proceedings.

Ghorbanian, K., Soltani, M. R., \& Manshadi, M. D. (2011). Experimental investigation on turbulence intensity reduction in subsonic wind tunnels. Aerospace science and Technology, 15(2), 137-147.

Hjärne, J., Löfdahl, L., \& Larsson, J. (2003). DESIGN OF A MODERN TESTFACILITY FOR LPT/OGV FLOWS. ASME Turbo Expo 2003, collocated with the 2003 Inter-national Joint Power Generation Conference, 137-145.

Leal González, F. A., \& Herández Cely, M. M. (2013). Estudio del potencial eólico y solar de cúcuta, norte de santander. Revista Colombiana de Tecnologías de Avanzada, 2(22), 27-33.

Leal González, F. A., \& Herández Cely, M. M. (2013). Estudio del potencial eólico y solar de cúcuta, norte de santander. Revista Colombiana de Tecnologías de Avanzada, 2(22), 27-33.

Kolmogorov, A. N. (1941). The local structure of turbulence in incompressible viscous fluid for very large Reynolds numbers. Paper presented at the Dokl. Akad. Nauk SSSR.

Leifsson, L., \& Koziel, S. (2015). Simulationdriven design of low-speed wind tunnel contraction. Journal of Computational Science, 7, 1-12.

Lindgren, B., \& Johansson, A. V. (2002). Design and evaluation of a low-speed wind-tunnel with expanding corners. Flow Facility Design and Experimental Studies of WallBounded Turbulent Shear-Flows, 63.

Mathew, J., Bahr, C., Carroll, B., Sheplak, M., \& Cattafesta, L. (2006). Design, fabrication, 
and characterization of an anechoic wind tunnel facility. University of Florida,

Menter, F. R., Kuntz, M., \& Langtry, R. (2003). Ten years of industrial experience with the SST turbulence model. Turbulence, heat and mass transfer, 4(1), 625-632.

Morel, T. (1975). Comprehensive design of axisymmetric wind tunnel contractions. Journal of Fluids Engineering, 97(2), 225-233.

Morel, T. (1977). Design of two-dimensional wind tunnel contractions. Journal of Fluids Engineering, 99(2), 371-377.

Rona, A., \& Soueid, H. (2010). Boundary layer trips for low Reynolds number wind tunnel tests.

Slangen, R. (2009). Experimental investigation of artificial boundary layer transition. Master of Science Thesis, TU Delft, vol, 82.

Watmuff, J. (1986). Wind tunnel contraction design. Paper presented at the Proceedings of 9th Australian Fluid Mechanics Conference.

Whitehead, L., Wu, L., \& Waters, M. (1951). Contracting ducts of finite length. The Aeronautical Quarterly, 2(4), 254-271. 